\title{
La orientación psicopedagógica en la práctica universitaria
}

Fecha de recepción : 12 de agosto de 2019 • Fecha de aceptación: 5 de septiembre de 2019 • Fecha de publicación: 10 de septiembre de 2019

Dra. C. Yaquelín Alfonso Moreira

Universidad Metropolitana, Ecuador. Universidad de Cienfuegos, Cuba

yalfonso@gmail.com

ORCID.0000-0002-6981-1966

Dra. C. María Guadalupe Valladares González

Universidad Metropolitana, Ecuador. Universidad de Cienfuegos, Cuba lupevalladaresg@gmail.com

ORCID.0000-0002-8813-1915

Dr. C. Miguel Pulido

Universidad Metropolitana, Ecuador. Universidad de Cienfuegos, Cuba miguelpulidocardena@umet.edu.cu ORCID.0000-0003-2055-8485

\section{Resumen}

Las concepciones para la orientación psicopedagógica deben asumirse como uno de los retos para el perfeccionamiento de la práctica universitaria, con énfasis en el nivel institucional y los colectivos de carrera. La identificación de esta problemática permitió analizar las reflexiones teóricas que configuran la posición metodológica de las acciones que se proponen en el artículo. El resultado de este proceso se va enriqueciendo de manera gradual a partir de la estrategia institucional, de manera que las tareas o actividades descansen en una concepción integral del proceso formativo en íntima relación con las demás áreas de servicio y de dirección. La experiencia desarrollada en los últimos dos semestres en la UMET permitió establecer el procedimiento que debe regir las acciones educativas para que estas impacten en la práctica universitaria hasta alcanzar el nivel de integración, coherencia y sistematicidad que se precisa en este tipo de orientación. Las concepciones que se presentan ponderan la responsabilidad en el trabajo metodológico y de vinculación, así como la importancia de especificar la intencionalidad de este proceso en el diseño y desarrollo del currículo. 


\begin{abstract}
Conceptions for psycho-pedagogical orientation should be assumed as one of the challenges for the improvement of university practice; with emphasis on the institutional level and career groups. The identification of this problem allowed to analyze the theoretical reflections that configure the methodological position of the actions proposed in the article. The result of this process has been gradually enriched from the institutional strategy so that the tasks or activities rest in a comprehensive conception of the training process in intimate relationship with the other areas of service and management. The experience developed in the last two semesters at the UMET, allowed to establish the procedure that should govern the educational actions so that they impact on university practice until reaching the level of integration, coherence and systematicity that is required in this type of orientation. The conceptions that are presented weigh the responsibility in the methodological and linking work; as well as the importance of specifying the intent of this process in the design and development of the curriculum.
\end{abstract}

Keywords: psycho-pedagogical orientation, career, training, strategies, curriculum. 


\section{Introducción}

La perspectiva de la orientación psicopedagógica es una exigencia del desarrollo científico pedagógico y, sobre todo, una condición necesaria para mejorar la formación de los estudiantes. Sin dudas, la orientación como vía para la formación cobra mayor significación y sentido en las prácticas universitarias, sobre todo al considerar el nivel de satisfacción y motivación de profesores y estudiantes por la actividad que en estos centros se realiza.

Conseguir este propósito deberá llevar a una conciliación de intereses y sobre todo a una comprensión mutua de los objetivos, los contenidos, los apoyos y los incentivos capaces de transformar los modos de actuación existentes. Esclarecer el alcance de estas proyecciones nos estimula a identificar como objetivo de este trabajo diseñar el procedimiento que direccione la orientación psicopedagógica desde la carrera, como nivel que favorece el protagonismo que deben tener los estudiantes universitarios en el momento histórico actual, así como el que deberán tener en el futuro, cuando se desenvuelvan como ciudadanos y profesionales.

Además, considerar las proyecciones de trabajo de la universidad que contextualiza el alcance de la formación e identifica la producción de conocimientos científicos, tecnologías en correspondencia con las demandas de la sociedad donde está enclavada. Además los objetivos de formación de las carreras que por su carácter pragmático definen en el perfil de egreso, las exigencias que deberán sustentar el currículo y las estrategias de formación que aportan al cumplimento de objetivos, se convierten en aspectos básicos para el desempeño, las necesidades y potencialidades de los recursos humanos que participan y le otorga intencionalidad a las influencias en una relación de formación dual donde ambos-el estudiante y el propio docente-son educados.

No obstante, los estudiantes, sujetos de la influencia educativa, se convierten en un primer referente para la gestión de la orientación psicopedagógica, sus necesidades y potencialidades se asumen como el eje de articulación de las influencias que deben ejercer todos los que participan en la práctica universitaria.

Es importante señalar, además, que los profesores universitarios, a partir de su modo de actuación, son los encargados de identificar y utilizar de manera armónica la participación de otros docentes, trabajadores no docentes y miembros de la comunidad, los cuales, por su liderazgo académico, científico, político o cultural, se convierten en la fuerza dinamizadora del proceso educativo en las instituciones de Educación Superior, concebidas estas como centros de educación abierta para todos. Todos estos se centran en la necesidad de buscar coherencia en el pensamiento, la proyección y la ejecución de las acciones, y están dirigidos a determinar las condiciones que deben regir la orientación psicopedagógica.

El resultado de este proceso se viene concretando de manera gradual a partir de la estrategia institucional de la UMET, de manera que las acciones dirigidas a resolver esta problemática descansan en una concepción integral del proceso de formación que enaltece el papel de la facultad y la carrera, en íntima articulación con las demás áreas que se involucran en la formación. Su identificación en esta institución permitió analizar las reflexiones teóricas que configuran la 
posición metodológica de la orientación psicopedagógica en la práctica universitaria y que pueden ser socializadas en las universidades ecuatorianas.

\section{Presupuestos teóricos}

La orientación psicopedagógica en la Universidad se concibe en función de lograr una formación de los estudiantes que les permita asumir los retos que la vida profesional les impone. En su concepción se integran todas las acciones intencionadas de los profesores y demás trabajadores, los cuales comparten las responsabilidades de articular de manera coherente su influencia de acuerdo con el sistema de formación y las actividades que se elaboran y ejecutan como parte de la estrategia institucional para lograr los objetivos del modelo del profesional de cada carrera.

Según el criterio de Martínez (2001), la orientación se concibe como un proceso asistencial en función de la actividad de estudio y su progreso. Por tanto, reducen su concepción a un solo elemento.

Atendiendo a ideas más avanzadas, Martínez de Codés (1998), Bisquerra (1988, 2005, 2009) y Repetto (1999) le atribuyen a la orientación los rasgos de ser un proceso de ayuda, de toma de decisiones. La concepción educativa de este autor, encuentra su especificidad en el carácter diagnóstico, preventivo, evolutivo y ecológico, dirigidos al desarrollo y al cambio optimizado del cliente y de su contexto.

Mientras, Rojas (2018) considera que la orientación debe ser un proceso continuo, cohe $\neg$ rente y colectivo, cualidades que enfoca a la toma de decisiones autónomas en términos de un éxito social y profesional, así como para ver las condiciones para el diseño y ejecución de sus proyectos a lo largo de toda la vida.

Desde nuestro criterio, la orientación psicopedagógica en las instituciones de Educación Superior es considerada como un complejo sistema integrador de los procesos formativos, formulado en correspondencia con los objetivos y contenidos para una etapa definida de la formación del estudiante y el grupo, a partir de las potencialidades, necesidades educativas y de manera cooperada y convenida.

Se integran todas las influencias y potencialidades de la sociedad en su conjunto para alcanzar las aspiraciones del modelo de persona integral que esta espera de acuerdo al marco ideológico definido, a partir de la propia orientación (individual y colectiva) y comunicación de los participantes. Particularmente en la formación de valores, la concepción del mundo y la preparación profesional, para insertarse plena y creadoramente en la sociedad, como expresión de la formación integral de la personalidad.

La orientación psicopedagógica en la universidad incluye todas las acciones que contribuyen al logro de los objetivos formativos y se basa en la contextualización del proyecto social e institucional, en cada carrera o espacio del currículo, asumiendo como eje principal la autoeducación. Esta condición explica que se concrete la relación de interdependencia con los procesos de formación y desarrollo integral del profesional y se compruebe en la práctica. 
Exige, además, un personal comprometido, implicado, reflexivo, creativo, que le permita ser flexible y la toma de decisiones, teniendo en cuenta las condiciones y características en que tiene lugar el proceso, ya que la actividad siempre contiene una dialéctica entre los que interactúan, se ajusta a los contextos de la teoría de la educación tanto como a la didáctica. Cuando se aborda lo referente a la estructura, principios y métodos, se aprecia su pertinencia, de manera que le son perfectamente ajustables los objetivos, las acciones, las operaciones, los contenidos, los fines, las metas, los motivos, los intereses, etc.

Durante el diagnóstico, entonces, se necesita la caracterización socio-psico-pedagógica del estudiante que permita valorar su desarrollo personal y profesional e indagar en las características del contexto en que se ha formado. Se enfatizará en los aprendizajes precedentes y la manera en que transfiere los saberes esenciales.

La identificación de las vivencias con respecto a la profesión, la vida universitaria y su preparación para asumir las nuevas exigencias, que supone el ingreso a una carrera universitaria, le permitirá diseñar la intervención educativa cuya naturaleza preventiva permite ajustar al cambio que es necesario introducir en el proceso y crear todas las oportunidades para que el estudiante pueda satisfacer las necesidades educativas desde sus potencialidades.

Esa consideración explica que las acciones, los dispositivos o redes de apoyo a la orientación psicopedagógica se sustenten en la perspectiva de la atención a la diversidad como premisa. En este caso la tutoría, los talleres, las actividades de discusión grupal resultan formas organizativas a priorizar en estos contextos.

Se asume entonces que la orientación psicopedagógica debe concebirse como parte del encargo científico y metodológico que se desarrolla para promover de manera preventiva la interrelación dialéctica entre la instrucción, la educación y el desarrollo de alternativas que permitan suprimir las manifestaciones o tendencias no compatibles con el modelo pedagógico universitario, el cual debe ser expresión de la cultura universitaria.

Desde la tradición pedagógica una de las alternativas de la orientación psicopedagógica más eficiente está precisamente en la influencia que ejercen los escenarios en los que tiene lugar el proceso educativo.

La institución universitaria tiene su fortaleza educativa en dos dimensiones: la curricular sugiere la intencionalidad educativa que sustenta el modo de actuación profesional, pero, además, por la dimensión extracurricular en la cual se ofrecen oportunidades de formación que incluyen todas las áreas de desarrollo personal, profesional y ciudadano.

La carrera, las escuelas, las empresas concretan vínculos con la actividad profesional y se modela la práctica asociada al ejercicio profesional. Por un lado, estas actividades complementan el aprendizaje teórico y práctico, pero suponen la inclusión de acciones que con la participación protagónica del estudiante puedan llevar a cabo la prevención, solución o transformación de situaciones o problemas que aparezcan en cualquier contexto donde interactúen. Por tanto, la valorización de las potencialidades para el perfeccionamiento de la actividad que realizan permiten 
responder así a los proyectos de vinculación o investigación, a partir de los saberes asimilados en otros escenarios o de los que se autogestiona el estudiante para su vínculo con la práctica.

Por tanto, las influencias que se orientan en cada contexto educativo se convierten en escenarios por excelencia que permiten concretar la responsabilidad social de la universidad y la transformación sociocultural que puede desarrollar el estudiante en los espacios comunitarios donde vive y trabaja.

Se trata aquí de orientar psicopedagógicamente al estudiante en su responsabilidad de ciudadano y joven universitario para que sea capaz de aportar a la sociedad, desde su participación protagónica, su propia transformación y la transformación social. También se destaca así la oportunidad de relacionarse con líderes y miembros de las comunidades, con los que comparte saberes y valores necesarios para apropiarse de los argumentos en el ejercicio de sus deberes ciudadanos.

Se precisa, entonces, una proyección educativa centrada en las metodologías que se promuevan desde cada escenario, el liderazgo, compromiso y apoyo de los directivos académicos y docentes de la institución universitaria para que estas sean más pertinentes y democráticas en las que se promueva la noción de lo eficaz en la orientación psicopedagógica, considerado en los planes estratégicos de la institución con una clara concreción en los planes individuales de los participantes.

Conseguir la participación e implicación de los docentes y de los estudiantes precisa llevar a cabo un proceso de esclarecimiento del momento histórico que se vive, de los encargos que se le asignan a la universidad, y articular desde todas las organizaciones la manera de optimizar recursos, actividades y contextos para satisfacer necesidades individuales, grupales, institucionales y sociales. La comprensión de esta unidad es básica para introducir el discurso educativo y concretarlo en la práctica.

La renovación de las metodologías para la orientación psicopedagógica debe suponer, entonces, una progresiva ampliación de espacios de asesoramiento y apoyo técnico a los estudiantes y al profesorado, sobre todo para aquellos que estén dispuestos a iniciar procesos innovadores en interés de ampliar la oferta educativa y ser capaces de responder a las demandas individuales, grupales y sociales que definen la función institucional. Por tanto, deberá trabajarse por alcanzar que a nivel institucional se logre un trabajo colaborativo y multidisciplinar que favorezca los niveles de preparación de los estudiantes y profesores para enfrentar la orientación psicopedagógica en los diferentes niveles de concreción en los que es posible obtener los objetivos formativos que también legitiman el perfil del egresado.

Por consiguiente, las acciones orientadoras deberán organizarse desde los procesos sustantivos y complementarse con otras actividades que confirmen la transformación educativa del estudiante como expresión del cruce de influencias entre los diversos escenarios y factores.

Las actividades investigativas, las de vinculación y las prácticas laborales, dada la unidad dialéctica en que se fundamentan, deberán tener una orientación transformadora que, centrada 
en el problema social de su profesión u objeto de estudio, genere tecnologías que garanticen la renovación e innovación de estos al introducir resultados de investigaciones anteriores o propuestas de soluciones que se generen desde la gestión participativa, o que sean generadas desde la aplicación del método científico y la utilización de la creatividad del estudiante o del grupo de estudiantes que participan en las actividades.

Para lograr establecer la orientación psicopedagógica desde la renovación de los métodos de gestión, en cuanto a información y resultados, precisan algunos aspectos claves, como el compromiso de los implicados con las estrategias y metodologías que se asuman. Esto implica eliminar el voluntarismo, proceder desde una planificación racional, objetiva, flexible y orientada a la práctica, asumir el diagnóstico de proceso y resultado como base para la toma de decisiones de manera que se adecúe y responda a cada contexto, grupo y sujeto de la orientación psicopedagógica en las universidades, diseñar estrategias dirigidas a la consecución de las metas con plazos fijados de antemano y en las que se indiquen los niveles de responsabilidad de los comprometidos, aprovechar al máximo los recursos de socialización de la experiencia y los resultados y convertirlos en referente metodológico para la acción perfeccionada de los demás, contar con dispositivos, espacios de trabajo y estimulación que se encarguen de impulsar, dinamizar, evaluar el proceso, estimular los resultados y los efectos de estos en el proceso de aprendizaje de los estudiantes, establecer el seguimiento y control de evaluación del proceso que permita identificar metodologías y experiencias positivas e incorporar al modelo educativo de la universidad al otorgar un carácter generalizador.

En este caso se precisa que el diseño de la orientación psicopedagógica de la carrera y su concreción en los años deberá atender desde la estrategia concebida para estos fines al siguiente procedimiento:

1. La carrera: determinación de prioridades de formación en su dimensión educativa:

- Perfiles de la carrera y diagnóstico.

- Identificación de las tareas que debe realizar el estudiante para complementar por semestre su formación cultural integral según los procesos sustantivos.

- Diseño de las acciones por parte de la carrera en función de la Política Afirmativa y el Modelo Educativo y Pedagógico de la Universidad.

- Definir las actividades, las tareas del estudiante y el profesor por procesos sustantivos y discretizar por semestres su prioridad.

- Establecer el compromiso del estudiante con su propia formación sintetizando las tareas según los procesos sustantivos contextualizado en la tutoría de acompañamiento.

- Diseño de las actividades de la estrategia de carrera para la etapa establecida.

2. A partir de esta estrategia de carrera los colectivos en grupos determinan las actividades por 
procesos sustantivos, los estudiantes y los profesores asignados a cada tarea, así como el cronograma.

- Socializar con estudiantes (intencionar y ajustar).

3. Evaluación del impacto de las acciones de la carrera y grupo a partir de los siguientes criterios: participación del 100\% de los estudiantes y profesores en las actividades, implicación en la dirección de los procesos, desarrollo de destrezas y valores asociada a la actividad profesional y de responsabilidad ciudadana, transferencia de los conocimientos curriculares y sociales, introducción de resultados de investigación científica de la universidad, socialización de resultados en eventos y publicaciones, reconocimiento social e institucional de los resultados del trabajo realizado.

- En este caso, el diseño de las tareas deberá cumplir los siguientes requisitos:

- complementar el diseño curricular según el perfil de las carreras,

- discretizar las tareas por grupo en la carrera y por etapas en el semestre,

- utilizar los proyectos y personalidades reconocidas en el trabajo científico cultural,

- incluir la evaluación cada tres meses,

- promover la introducción de los resultados de investigación,

- estimular la participación del estudiante y el liderazgo para el cambio (actor social del cambio),

- centrar la transformación de modos de actuación, la orientación multidisciplinar,

- articular objetivos de trabajo,

- recopilar evidencias.

Por tanto, las tareas deben ser personalizadas y declarar el impacto social, estar basadas en la metodología de la gestión participativa y el trabajo en colaborativo que promuevan la implicación, ser medibles en corto y mediano plazo, determinar el seguimiento y control, además de asegurar la socialización y la evaluación del impacto.

Estas ideas explican que la renovación metodológica de la orientación psicopedagógica en la universidad supone que los profesores no se limiten a la reproducción de los métodos educativos que utilizaron con ellos, y los estudiantes han de ir tomando conciencia de que forman parte importante del proceso, debemos aprender a contextualizar, a protagonizar los cambios y transformar juntos la manera de educar en los centros universitarios, procurando la sostenibilidad de los resultados. 


\section{Materiales y métodos}

Los materiales utilizados para la investigación fueron el currículo de formación del Licenciado de Educación Inicial y Desarrollo del Talento Infantil para los estudiantes de la oferta académica del periodo 47, en las asignaturas declaradas en este estudio; así como, el Currículo de Educación Inicial para el contexto ecuatoriano vigente desde el 2014. En el primero, se describen los contendidos para la propuesta psicopedagógica incluyendo la experiencia práctica para su despliegue en los centros de Educación Inicial durante la práctica preprofesional, el futuro desempeño y en el proyecto de vinculación con la sociedad "Orientación psicopedagógica en la educación familiar formal y no formal". Se alega además, su contextualización a partir de los ámbitos y destrezas que se deben formar en los niños/as en los dos subniveles de Educación Inicial.

\section{Métodos}

Los principales métodos utilizados fueron el análisis documental aplicado a ambos currículos con la técnica de análisis de contenido, con el que se identificó el contenido del proceso de formación del Licenciado en Educación Inicial y su articulación con los ejes, ámbitos y destrezas del subnivel 2 de Educación Inicial para su aplicación en la experimentación de la práctica pedagógica, constituyendo este otro de los métodos basados en la relatoría de las experiencias prácticas donde se aplica este proyecto. Se aplicó además el método de observación de los participantes en la exposición de dichas experiencias.

\section{La proyección y los resultados de la experiencia desde las asignaturas relacionadas con el eje de expresión y comunicación}

En las concepciones pedagógicas y didácticas actuales se destaca la importancia de los ejes de contenido como propuesta renovadora en el proceso de formación para integrar saberes de diferentes disciplinas y asignaturas del currículo escolar.

En tal sentido Botero (2008) considera que los ejes son instrumentos que atraviesan la totalidad del currículo y en particular la totalidad de las áreas del conocimiento de las disciplinas y los temas con la finalidad de revisar las estrategias aplicadas tradicionalmente en el aula al incorporar al currículo, en todos sus niveles, una educación significativa para el estudiante, a partir de la conexión de las áreas del saber. Desde esta perspectiva, los ejes transversales constituyen grandes temáticas que deben ser atendidas en toda la proyección de la maya curricular, con actividades concretas integradas al desarrollo de las habilidades en el campo de la profesión que se forman los estudiantes y abrir las fronteras de lo disciplinar y al ubicar como núcleo para el encuentro dialógico en problemáticas o temas curriculares.

En el contexto del trabajo se utiliza la concepción de eje como un recurso didáctico para la innovación educativa con la finalidad de crear condiciones favorables para proporcionar a los estudiantes una mayor formación académica y profesional sustentada en determinados 
contenidos del currículo. Cómo toda propuesta estos ejes deben ser abiertos y flexibles, para que tengan la posibilidad de incorporar nuevos contenidos; no son responsabilidad de una asignatura en particular; si no, que integra desde una asignatura varias de ellas en la maya curricular desde el eje articulador expresión y comunicación en correspondencia con lo planteado en el currículo de Educación Inicial para el contexto ecuatoriano estructurado en ejes de desarrollo y de aprendizaje.

Este currículo es uno de los espacios que integran un conjunto de aspectos relacionados con el proceso de formación de niñas y niños con el propósito de organizar los objetivos de aprendizaje y las destrezas en cada uno de los subniveles de Educación Inicial. Esta concepción debe articularse con el proceso de formación para su desempeño profesional. En tal sentido, en la carrera de Educación Inicial, como parte de la oferta académica, debe quedar clarificado la relación entre formación y práctica docente, teniendo en cuenta los ejes del currículo de Educación Inicial y las materias que tributan a él.

Este currículo está estructurado por ejes de aprendizaje, entre ellos el eje de Expresión y Comunicación. En el mencionado currículo se precisa que en este eje "se consolidan procesos para desarrollar la capacidad comunicativa y expresiva de los niños, empleando las manifestaciones de diversos lenguajes y lenguas, como medios de exteriorización de sus pensamientos, actitudes, experiencias y emociones que les permitan relacionarse e interactuar positivamente con los demás" (Ministerio de Educación. Currículo de Educación Inicial, 2014, p. 19).

De este eje se derivan los ámbitos de manifestación del lenguaje verbal y no verbal, así como de exploración del cuerpo y de motricidad, que corresponde al subnivel 1; mientras que para el subnivel 2 los ámbitos son: compresión y expresión oral y escrita, compresión y expresión artística y expresión corporal, entendidos como espacios curriculares más específicos que se derivan de los ejes de desarrollo y aprendizaje que identifican, secuencia y organizan los objetivos de aprendizaje y las destrezas en cada uno de los subniveles de Educación Inicial (Currículo de Educación Inicial, 2014, p. 17).

En las asignaturas se determinan las prioridades de formación en su dimensión educativa en los programas de estudio de asignaturas, las que se muestran a continuación.

En el eje de comunicación y expresión, en el Currículo de Educación Inicial, se describen como contenidos:

- La comunicación y el lenguaje: enfoque sociocultural

- Métodos, técnicas, recursos didácticos y lúdicos

- La expresión artística

- La narrativa literaria y corporal

- Lectura y comprensión de textos escritos y orales 
- La argumentación y sus elementos básicos

- Reglas y normas de redacción

En relación con lo apuntado, las asignaturas de la carrera relacionadas con el eje de expresión y comunicación -Psicolingüística, Pedagogía del Lenguaje, Iniciación de la lectoescritura, Expresión Artística, Plástica y Expresión Dramática y Corporal - hacen notar la orientación psicopedagógica del contenido, el significado, el sentido que adquiere para el sujeto y para la práctica social que tiene para el estudiante el aprendizaje, para que pueda luego hacer transferencia y uso desde otros escenarios, mediante la socialización de sus aprendizajes a través de actividades lúdicas como plataforma trasversal al currículo, o la intervención especializada en grupos de investigación, de vinculación o en trabajos autónomos que se le asignen por encargo a los estudiantes.

1. Las asignaturas: determinación de prioridades de formación en su dimensión educativa:

- Programa de estudio de asignaturas y diagnóstico.

- Identificación de las tareas que debe realizar el estudiante para complementar por semestre su formación cultural integral según el eje de expresión y comunicación y los componentes de cada asignatura.

- Diseño de las acciones de la asignatura.

- Definir las actividades, tareas del estudiante y el profesor según el eje de expresión y comunicación y discretizar por temas su prioridad.

- Establecer el compromiso del estudiante con su propia formación sintetizando las tareas según las relaciones entre los componentes de las asignaturas y el eje de expresión y comunicación.

- Diseño de las actividades de cada asignatura para la etapa establecida.

2. A partir de esta estrategia, las asignaturas articulan sus contenidos y los colectivos por grupos: determinan actividades, los estudiantes y profesores, elaboran el cronograma del proyecto integrador.

- Socializar con estudiantes (intencionar y ajustar)

3. Evaluación del impacto de las acciones a partir de los siguientes criterios: participación del $100 \%$ de los estudiantes y profesores en las actividades, implicación en la dirección de los procesos, desarrollo de destrezas y de valores asociados a la actividad profesional, desarrollo de responsabilidad ciudadana, transferencia de los conocimientos curriculares y sociales, introducción de resultados de investigación científica de la universidad, socialización de resultados en eventos y publicaciones, reconocimiento social e institucional de los resultados del trabajo realizado. 
Se identificaron las tareas que debe realizar cada estudiante para complementar por semestre su formación cultural integral según el eje de expresión y comunicación y los componentes de cada asignatura, y los recursos didácticos para la adquisición y desarrollo del lenguaje resultaron ser el elemento integrador.

Se definieron las actividades y tareas del estudiante y el profesor según el eje de expresión y comunicación para discretizar por temas su prioridad y el resultado fue elaborar un ensayo académico para argumentar las ideas relacionadas con el eje y los contenidos de las asignaturas.

Se estableció el compromiso de los estudiantes con su propia formación sintetizando las tareas según las ideas que ellos sugerían y las relaciones entre los componentes de las asignaturas y el eje de expresión y comunicación.

Se diseñaron entre todos las actividades de cada asignatura para la etapa establecida; las asignaturas articularon sus contenidos y los colectivos por grupos determinaron las actividades y elaboraron el cronograma del proyecto integrador.

Se socializó con estudiantes, se intencionó y ajustaron con los estudiantes los tipos de recursos y sus argumentos.

Se evaluó un impacto de la estrategia a corto plazo que nos permitió identificar que participaron el $100 \%$ de los estudiantes y profesores en las actividades, se implicaron en la dirección de los procesos, se desarrollaron destrezas y valores asociados a la actividad profesional y de responsabilidad ciudadana, se transfirieron los conocimientos curriculares y sociales, se introdujeron los resultados de investigación científica de la universidad, se socializaron los resultados en eventos y publicaciones y hubo un reconocimiento social e institucional de los resultados del trabajo realizado.

Dichos resultados expresan la articulación de los contenidos tratados en el proceso de formación y su contextualización práctica en las diferentes formas de evaluación sistemática, parcial y final, esta última como forma de culminación de las materias en trabajos integradores.

Entre los resultados más notables se distinguen los temas relacionados con el lenguaje y la comunicación, proponiéndose como recursos didácticos el teléfono, las botellas sensoriales, la trompeta, el abecedario, el bingo de vocales, el rompecabezas, el parchís, el tablero mágico, los títeres, la rueda de colores, los dados de la suerte, la ruleta de los colores y la de los sentimientos, el delantal cuenta cuentos, el libro de las emociones y el del cuerpo humano, el franelógrafo, la caja misteriosa, el rincón del arte, entre otros que se muestran en las imágenes que aparecen en el anexo N. ${ }^{\circ} 1$.

\section{Discusión}

La orientación psicopedagógica ha sido una necesidad en el ámbito educativo y pedagógico desde el siglo XX, que se ha ido perfeccionado a partir de la renovación de sus enfoques y estilos 
de aplicación acorde con las exigencias de la calidad educativa en general y del proceso de enseñanza-aprendizaje en particular.

Diversos estudios dan cuenta de este problema de investigación; sin embargo, resultados similares pueden localizarse en diversas investigaciones, como por ejemplo en "Los ejes transversales como instrumento pedagógico para la formación de valores” de Botero (2008), quien centra su atención en la formación de valores, concepción que se relaciona en la propuesta con la dimensión socioafectiva.

En otro orden, similar propuesta para el proceso de formación se localiza en el trabajo de Nieto Almeida (2005) Modelo de superación profesional para el perfeccionamiento de las competencias profesionales en la orientación psicopedagógica con profesores de los ISP, sin llegar a su contextualización en el currículo del desempeño en la práctica.

De producción más reciente y con resultados similares, se localizan dos trabajos de titulación en la carrera de Educación Inicial y Desarrollo del Talento Infantil en la UMET, de las autoras Alvear (2019) y Sosa (2019), quienes realizan propuestas para su aplicación práctica en el ejercicio de la profesión.

La orientación psicopedagógica se concibió desde una concepción estratégica que se insertó en la renovación de la universidad al sustentar nuevos estilos y métodos de trabajo, al superar la influencia centrada en el aula y promover como alternativa las dimensiones y los procesos sustantivos, considerando como determinante la actuación coordinada y cooperativa de todos y orientando las diversas acciones hacia la diferencia, lo cual supone centrarse en el estudiante, ponerse en función de sus necesidades, crear un ambiente orientador que promueva el compromiso y apoyo de los que participan al organizar, ejecutar y evaluar la estrategia educativa según el nivel de trabajo metodológico o de dirección que represente. El procedimiento propuesto incluyó las acciones, las operaciones de las acciones educativas de la carrera, el grupo y la evaluación, atendiendo a criterios y requisitos que permitieran evaluar el impacto de la orientación psicopedagógica. 


\section{Referencias bibliográficas}

Alvear, D. E. (2019). La orientación familiar para estimular el desarrollo socioafectivo en los niños y niñas de 2 a 3 años del Guagua Centro La Bota. Unidad Curricular para la obtención del Título Desarrollo del Talento infantil. Inédito, UMET. Quito.

Bisquerra, R. (2009). La orientación como proceso educativo. Barcelona, España: Morata.

Bisquerra, R. (2005). Marco conceptual de la orientación educativa. Revista mexicana de orientación educativa, 3(6), 2-7. Recuperado de http://www.remo.ws/revistas/remo-6.pdf

Bisquerra, R. (1988). Modelos de orientación e intervención. Barcelona, España: Praxis.

Botero, C. A. (2008). Los ejes transversales como instrumento pedagógico para la formación de valores. Revista Iberoamericana de Educación, 45(2). Recuperado de https://www.researchgate.net/publication/28203272_ Los_ejes_transversales_como_instrumento_pedagogico_para_la_formacion_de_valores

Martínez, O. (2001). Orientación educativa en la Escuela Básica. Una propuesta de cambio. Caracas, Venezuela: UNA.

Martínez de Codès, M. (1998). La Orientación Escolar. Madrid, España: Sáenz y Torres.

Ministerio de Educación (2014). Currículo de Educación Inicial, Ecuador.

Nieto, L. E. (2005). Modelo de superación profesional para el perfeccionamiento de las competencias profesionales en la orientación psicopedagógica con profesores de los ISP (tesis doctoral). ISP, Santa Clara, Cuba.

Repetto, E. (1999). Tu futuro profesional. Tomo III. Libro del alumno. Ciencias de la educación preescolar y especial. Madrid, España: Pardiñas.

Sosa, M. (2019). Estimulación del lenguaje en los niños/as de 3 a 4 años del Centro de Desarrollo Infantil Juan Enrique Pestalozzi. Unidad Curricular para la obtención del Título Desarrollo del Talento infantil. Inédito, UMET. Quito.

Valladares, A. L. (27 de febrero de 2018). La orientación psicopedagógica en el contexto educativo. Quito: Universo Sur. Recuperado de Concepto de los ejes transversales, sus objetivos y su aplicación: http://www. webscolar.com/concepto-de-los-ejes-transversales-sus-objetivos-y-su-aplicacion 


\section{Anexos}

Anexo N. ${ }^{\circ} 1$.

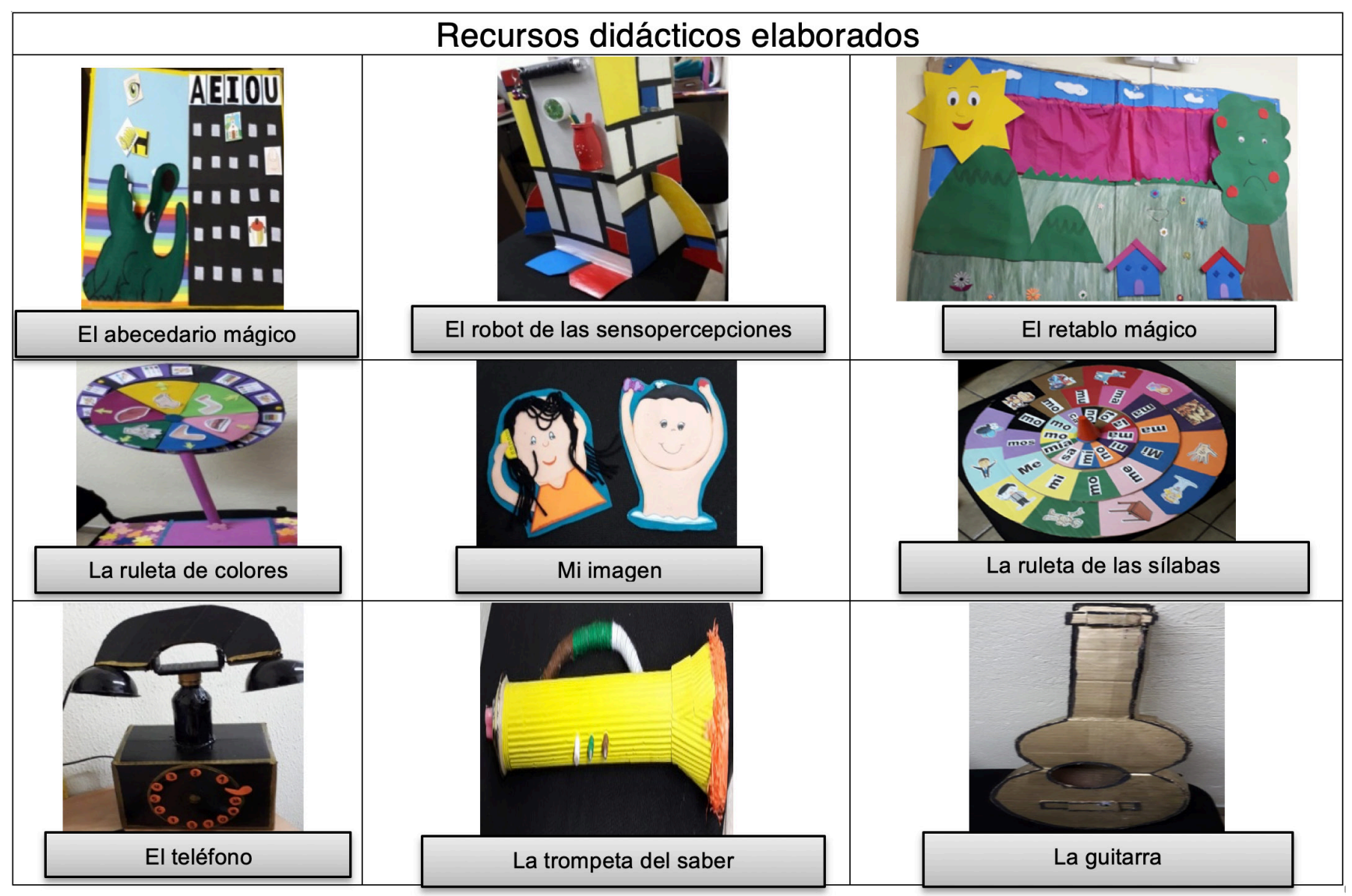


